\title{
Effect of Substance P on type II alveolar epithelial cells exposed to hyperoxia and its regulation of the Sonic hedgehog signaling pathway
}

\author{
CONG LIU, LIN YANG, HONGXING DANG, FANG FANG and FENG XU
}

\begin{abstract}
Ministry of Education Key Laboratory of Child Development and Disorders, Key Laboratory of Pediatrics in Chongqing, CSTC2009CA5002, Chongqing International Science and Technology Cooperation Center for Child Development and Disorders, Pediatric Intensive Care Unit, Children's Hospital of Chongqing

Medical University, Chongqing 400014, P.R. China
\end{abstract}

Received September 13, 2013; Accepted March 17, 2014

DOI: $10.3892 / \mathrm{mmr} .2014 .2330$

\begin{abstract}
Oxidative stress injury and cell death in alveolar epithelial cells may lead to abnormal repair, further resulting in acute and chronic pulmonary diseases. Substance P (SP) has multiple biological activities. The Sonic hedgehog (SHH) pathway is important in lung development and decreasing epithelial injury. To investigate the effects of SP on alveolar epithelial type II cells (AEC IIs), AEC IIs were exposed to 95\% oxygen and the SHH signaling pathway was examined. Primary AEC IIs were isolated and purified from premature rats. The cells were divided into four groups: The air ( $21 \%$ oxygen) group, hyperoxia (95\% oxygen) group, hyperoxia + SP group and hyperoxia $+\mathrm{SP}+\mathrm{L} 703.606$ group. The activity of AEC IIs was examined using a 3-(4,5-dimethylthiazol-2-yl)-2,5-diphenyltetrazolium bromide assay. The apoptotic rate of AEC IIs was analyzed by flow cytometry. The oxidative damage was evaluated by flow cytometry and reactive oxygen species (ROS) were detected using a 2',7'-dichlorodihydrofluorescein diacetate probe. Quantitative polymerase chain reaction and western blotting were used to detect the mRNA and protein expression of the SHH signaling molecule Smoothened (SMO). The results demonstrated that exposure to $95 \%$ oxygen for $24 \mathrm{~h}$ significantly increased the level of ROS, contributed to apoptosis and markedly decreased the proliferation of AEC IIs. Compared with hyperoxia exposure, SP treatment decreased the level of ROS, reduced AEC II apoptosis and improved the cell survival sequentially. SMO was found to be expressed in AEC IIs and its expression increased when the cells were in hyperoxic condi-
\end{abstract}

Correspondence to: Professor Feng $\mathrm{Xu}$, Pediatric Intensive Care Unit, Children's Hospital of Chongqing Medical University, 136 Zhongshan Second Road, Yuzhong, Chongqing 400014, P.R. China

E-mail: liucong0757@yeah.net

Key words: hyperoxia, alveolar epithelial type II cell, substance P, L703.606, Sonic hedgehog signaling pathway tions. These effects were enhanced by treatment with SP, which was able to significantly increase the expression of SMO. The aforementioned protective effect was weakened following treatment with L703.606. These findings suggested that SP was a protective regulatory factor that was able to decrease the hyperoxia-induced cell injury and death, and improve the survival of AEC IIs exposed to hyperoxia, which may be associated with the activation of the SHH signaling pathway.

\section{Introduction}

Prolonged exposure to high concentrations of oxygen ( $>90 \%$ oxygen) can lead to either acute or chronic lung injury in former premature infants. It leads to the production of free oxygen radicals that exceed the cell defense capacity, leading to inflammation, cell damage and gene overexpression that may result in necrosis and apoptosis (1).

The alveolar epithelium is the major target of oxidant injury (2) and repair of the injury depends on the ability of its stem cells, the alveolar epithelial type II cells, which are able to spread and proliferate. Alveolar epithelial type II cells (AEC IIs) can spread, proliferate and are important in the repair process of the alveolar epithelium. (3).

Substance P (SP) is widely distributed in the airway endothelial cell layer. It was revealed that SP is able to trigger an exuberant neuroinflammatory response and regulate proliferation, migration and differentiation of the impaired cells $(4,5)$. However, the molecular mechanisms that regulate the activities of AEC IIs and attenuate oxidative stress injury are poorly understood. It has been reported that the Sonic hedgehog ( $\mathrm{SHH}$ ) pathway is critical in lung morphogenesis and organogenesis $(6,7)$. SHH signaling proteins, including SHH, Patched, Smoothened (SMO) and Gli are important in a variety of processes, including embryogenesis, tissue repair and wound healing (8-10).

The aim of the present study was to investigate the effects of SP on primary AEC IIs of premature rats exposed to hyperoxia and its regulation of the SHH signaling pathway. In view of this, the present study aimed to elucidate the associated regulatory mechanism of SP in the damage to AEC IIs exposed to hyperoxia. 


\section{Materials and methods}

Experimental animals. The 180-200 g specific pathogen-free Sprague-Dawley (SD) rats were obtained for the present study from the Experimental Animal Center of Chongqing Medical University (Chongqing, China). All the experiments were conducted in accordance with the National Guidelines for the Care and Use of Laboratory Animals. The experiment was approved by the Ethics Committee of Chongqing Medical University (Chongqing, China).

Materials. SP was provided by Abcam (Cambridge, MA, USA) and L703.606, a selective NK1R antagonist, was provided by Sigma (St. Louis, MO, USA). The Annexin V fluorescein isothiocyanate (FITC)-labeled apoptosis kit was provided by Kaiji Biotechnology Co., Ltd. (Nanjing, China). SMO polyclonal antibody was purchased from Abbiotec (San Diego, CA, USA) and the reactive oxygen species kit was purchased from Beyotime Biotechnology Co., Ltd. (Shanghai, China).

Culture and isolation of premature rats. Healthy adult SD rats were raised in a cage with a gender ratio of 1:1. Pregnancy was confirmed if a vaginal plug was observed on the second morning following the mixed raising. Uterine-incision delivery was employed to remove the premature rats from the pregnant rats on day 19 (22 days for full term) and fetal lungs were isolated. AEC IIs were isolated through digestion with $0.25 \%$ trypsin and $0.04 \mathrm{mg} / \mathrm{ml}$ DNAse for $20 \mathrm{~min}$ at $37^{\circ} \mathrm{C}, 0.1 \%$ collagenase for $15 \mathrm{~min}$ at $37^{\circ} \mathrm{C}$ and centrifugation at $200 \mathrm{x} \mathrm{g}$ for $5 \mathrm{~min}$. Subsequently, the isolated cells were stained with modified Papanicolaou stains as previously described (11) and observed under a transmission electron microscope (Hitachi, Ltd., Tokyo, Japan).

Establishment of the cellular oxidative model. Following culturing for $24 \mathrm{~h}$, the isolated and purified cells were treated with air ( $21 \%$ oxygen), hyperoxia ( $95 \%$ oxygen), SP + hyperoxia or SP + L703.606 + hyperoxia. The cells were exposed to air and hyperoxia for $24 \mathrm{~h}$ in a closed oxygen chamber; $1 \times 10^{-8} \mathrm{~mol} / 1 \mathrm{SP}$ and $1 \times 10^{-7} \mathrm{~mol} / 1 \mathrm{~L} 703.606$ were added in advance. Then, the cells were exposed to air and hyperoxia for $24 \mathrm{~h}$. The cells were collected $24 \mathrm{~h}$ after exposure.

3-(4,5-dimethylthiazol-2-yl)-2,5-diphenyltetrazolium brom ide (MTT) cellular proliferation assay. The proliferation of treated AEC IIs was detected using an MTT detection kit (Amresco LLC, Solon, OH, USA) according to the manufacturer's instructions.

Apoptosis assay. The cellular apoptosis and necrosis were detected using an Annexin V-FITC apoptosis detection kit on the basis of Annexin $\mathrm{V}$ and propidium iodide staining. Apoptosis was assayed by flow cytometry (BD Biosciences, Franklin Lakes, NJ, USA).

Oxidation assay. To evaluate the damage caused by oxygen, reactive oxygen species (ROS) were measured using a flow cytometer (BD Biosciences, Franklin Lakes, NJ, USA) according to the manufacturer's instructions.
Quantitative polymerase chain reaction ( $q P C R$ ). $\mathrm{qPCR}$ was performed using the SYBR-Green real-time PCR method. Total RNA was extracted from AEC IIs. qPCR was performed using an FTC2000 PCR instrument (Funglyn Biotech Inc., Toronto, Ontario, Canada) using two-stage program parameters provided by the manufacturer, as follows: $4 \mathrm{~min}$ at $94^{\circ} \mathrm{C}$ and then 35 cycles of $20 \mathrm{sec}$ at $94^{\circ} \mathrm{C}, 30 \mathrm{sec}$ at $60^{\circ} \mathrm{C}$ and $30 \mathrm{sec}$ at $70^{\circ} \mathrm{C}$. The specificity of the produced amplification product was confirmed by the examination of dissociation reaction plots. A distinct single peak indicated that a single DNA sequence was amplified during PCR. PCR products were run on $2 \%$ agarose gels to confirm that the correct molecular sizes were presented. Each sample was assessed in triplicate and the samples obtained from three independent experiments were used for the analysis of relative gene expression using the $2^{-\Delta \Delta \mathrm{Ct}}$ method. The following primers were used for qPCR: Forward: 5'-CCC ATC TAT GAG GGT TAC GC-3' and reverse: 5'-TTT AAT GTC ACG CAC GAT TTC-3' for glyceraldehyde-3-phosphate dehydrogenase; and forward: 5'-ACC TCC AGC GAG ACC CTA TC-3' and reverse: 5'-TGA GGA CGA AGG GGA GTG AC-3' for SMO.

Western blot analysis. Total cellular proteins were calculated using the bicinchoninic acid protein assay and proteins $(50 \mu \mathrm{g})$ from each sample were loaded onto a $10 \%$ SDS/polyacrylamide gel and electrophoretically transferred onto polyvinylidene fluoride membranes. The membranes were inhibited in Tris-buffered saline with Tween-20 (TBST) containing $0.05 \%$ Tween-20/5\% non-fat dried milk for $1 \mathrm{~h}$, rinsed, and incubated with the appropriate smo polyclonal antibody (Santa Cruz Biotechnology, Inc., Santa Cruz, CA, USA) overnight at $4^{\circ} \mathrm{C}$. Following washing in TBST, the membranes were incubated with horseradish peroxidase secondary antibody (GenScript Ltd., Piscataway, NJ, USA) for $1.5 \mathrm{~h}$ at room temperature, followed by three washes in TBS. The immunoreactive proteins were visualized with peroxidase and an enhanced chemiluminescence system (ECL kit; Pierce Biotechnology, Inc., Rockford, IL, USA).

Statistical analysis. All the data are expressed as the mean \pm standard deviation. The statistical significance of the differences between the means of the groups was determined by one-way analysis of variance or two-tailed Student's t-tests. $\mathrm{P}<0.05$ was considered to indicate a statistically significant difference.

\section{Results}

Effects of SP on cell growth and apoptosis of AEC IIs following exposure to hyperoxia. Following exposure to hyperoxia for $24 \mathrm{~h}$ and compared with the air treatment, AEC II proliferation markedly decreased and the percentage of apoptotic cells markedly increased (Figs. 1-2). Furthermore, it was revealed that following SP treatment, the proliferation activity increased compared with simple exposure to hyperoxia, which was able to be reversed by L703.606 (Fig. 1). Accordingly, the number of apoptotic cells decreased markedly following treatment with SP when compared with that in the hyperoxia group. This decrease was inhibited by L703.606 (Fig. 2). 


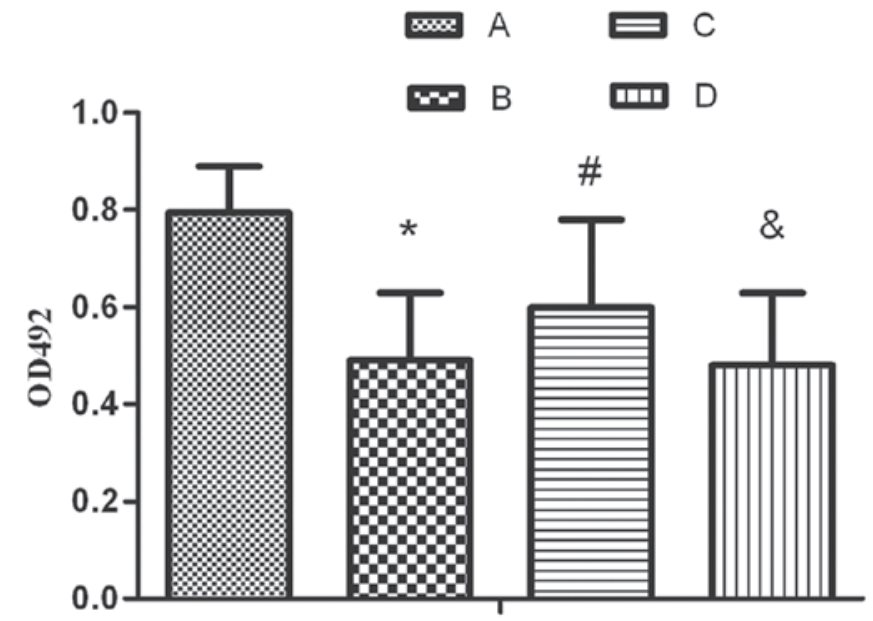

Figure 1. Effect of hyperoxia and SP on cell growth. The proliferation of alveolar epithelial type II cells was markedly suppressed $24 \mathrm{~h}$ after exposure to hyperoxia, compared with exposure to air. The proliferation activity increased following SP + hyperoxia treatment. However, the effect of SP on the proliferation activity was inhibited by L703.606. A, air group; B, hyperoxia group, $\mathrm{C}$, hyperoxia + SP group; D, hyperoxia + SP + L703.606 group; SP, substance P.; OD, optical density. ${ }^{~} \mathrm{P}<0.05$ vs. A; ${ }^{*} \mathrm{P}<0.05$ vs. B and ${ }^{\circledR} \mathrm{P}<0.05$ vs. C.

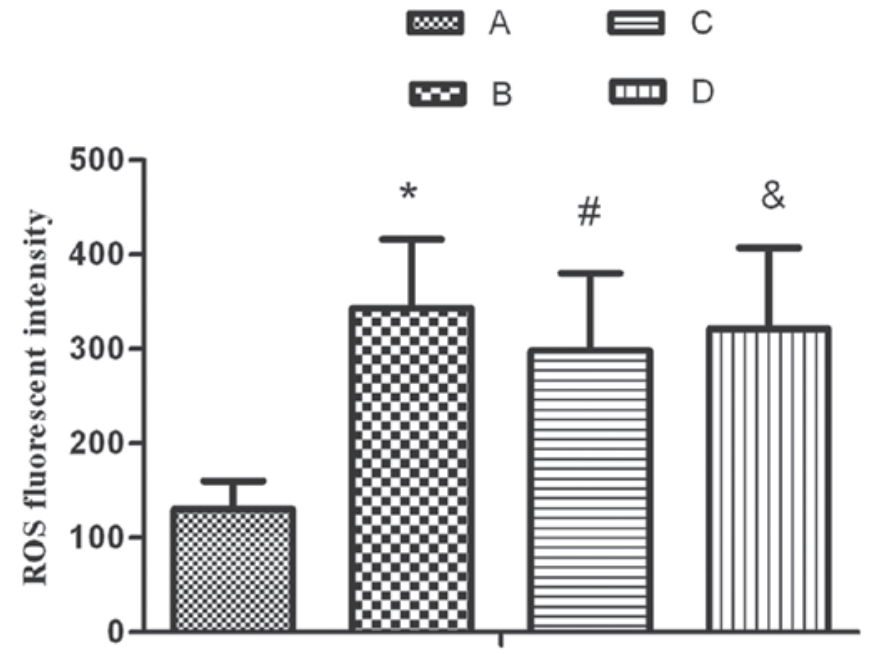

Figure 3. Effect of hyperoxia and SP on cell oxidative damage. ROS markedly increased $24 \mathrm{~h}$ after exposure to hyperoxia. The additional SP significantly decreased the levels of ROS in alveolar epithelial type II cells. However, the effect of SP on the level of ROS was reversed by L703.606. A, air group; $\mathrm{B}$, hyperoxia group, $\mathrm{C}$, hyperoxia $+\mathrm{SP}$ group; D, hyperoxia $+\mathrm{SP}+\mathrm{L} 703.606$ group); SP, substance $\mathrm{P}$; ROS, reactive oxygen species. " $\mathrm{P}<0.05$ vs. A; ${ }^{"} \mathrm{P}<0.05$ vs. $\mathrm{B}$ and ${ }^{\&} \mathrm{P}<0.05$ vs. $\mathrm{C}$.
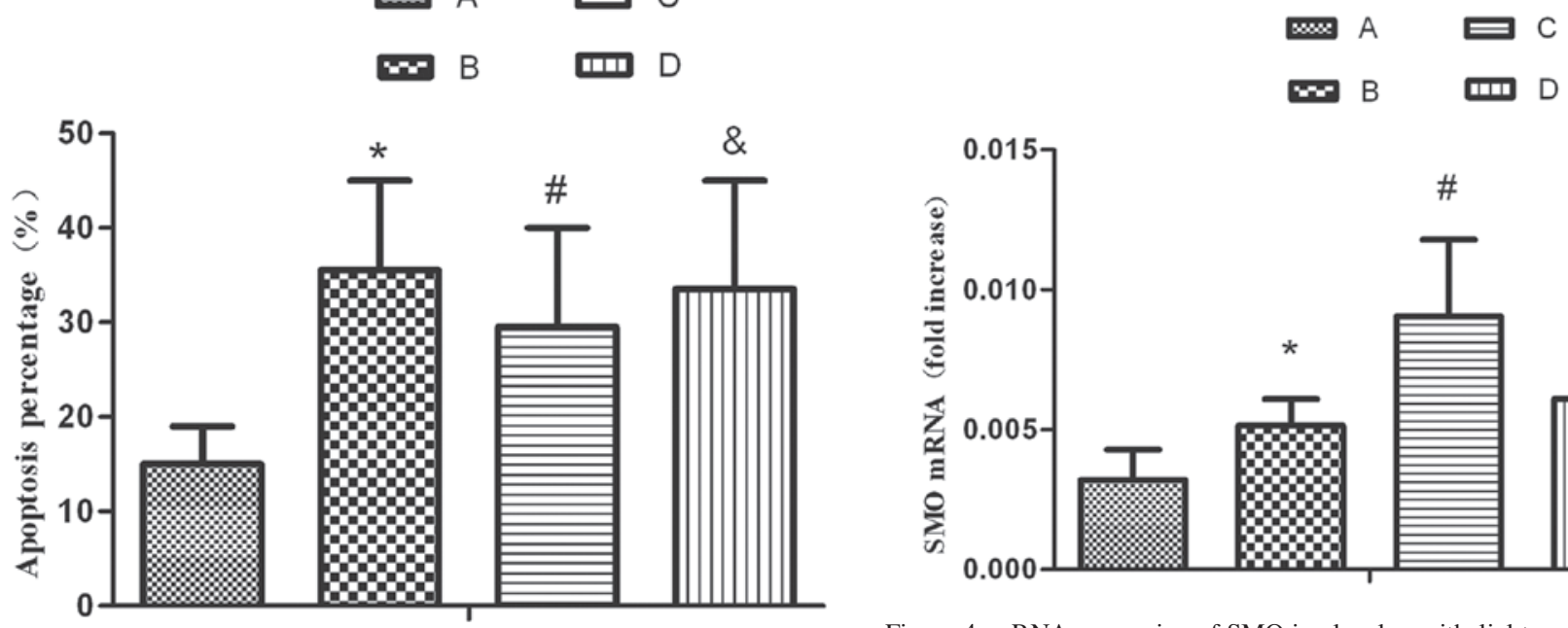

Figure 2. Effect of hyperoxia and SP on cell apoptosis. The percentage of apoptotic AEC IIs markedly increased $24 \mathrm{~h}$ after exposure to hyperoxia. The additional SP significantly suppressed the apoptosis of AEC IIs. However, the effect of SP on apoptosis was suppressed by L703.606. A, air group; $\mathrm{B}$, hyperoxia group, $\mathrm{C}$, hyperoxia $+\mathrm{SP}$ group; $\mathrm{D}$, hyperoxia $+\mathrm{SP}+\mathrm{L} 703.606$ group); SP, substance P; AEC IIs, alveolar epithelial type II cells. " $\mathrm{P}<0.05$ vs. $\mathrm{A} ;{ }^{\#} \mathrm{P}<0.05$ vs. $\mathrm{B}$ and ${ }^{\&} \mathrm{P}<0.05$ vs. $\mathrm{C}$.

Effect of SP on the oxidative damage of AEC IIs following exposure to hyperoxia. The results demonstrated that the content of ROS significantly increased upon treatment with 95\% oxygen for $24 \mathrm{~h}$. SP markedly inhibited the increase in ROS; however, L703.606 reversed the ROS level to a similar level as that in the hyperoxia treatment group, as shown in Fig. 3.

mRNA levels of SMO in AEC II cells are activated by hyperoxia and enhanced by SP treatment. qPCR revealed that SMO mRNA was expressed in normal AEC IIs. The mRNA expression of SMO in the hyperoxia-exposed group significantly increased compared with the air-exposed group. By contrast, the expression of SMO in the hyperoxia-exposed group was elevated by stimulation with SP. SP was able to significantly increase the expression of SMO. The aforementioned upregulation effect was attenuated following treatment with L703.606 (Fig. 4).

Protein levels of SMO in AEC II cells are activated by hyperoxia and enhanced by SP treatment. Western blot analysis revealed that SMO proteins were expressed in normal AEC IIs. The protein expression of SMO in the hyperoxia-exposed group was significantly increased compared with that in the air-exposed group. In addition, the expres- 

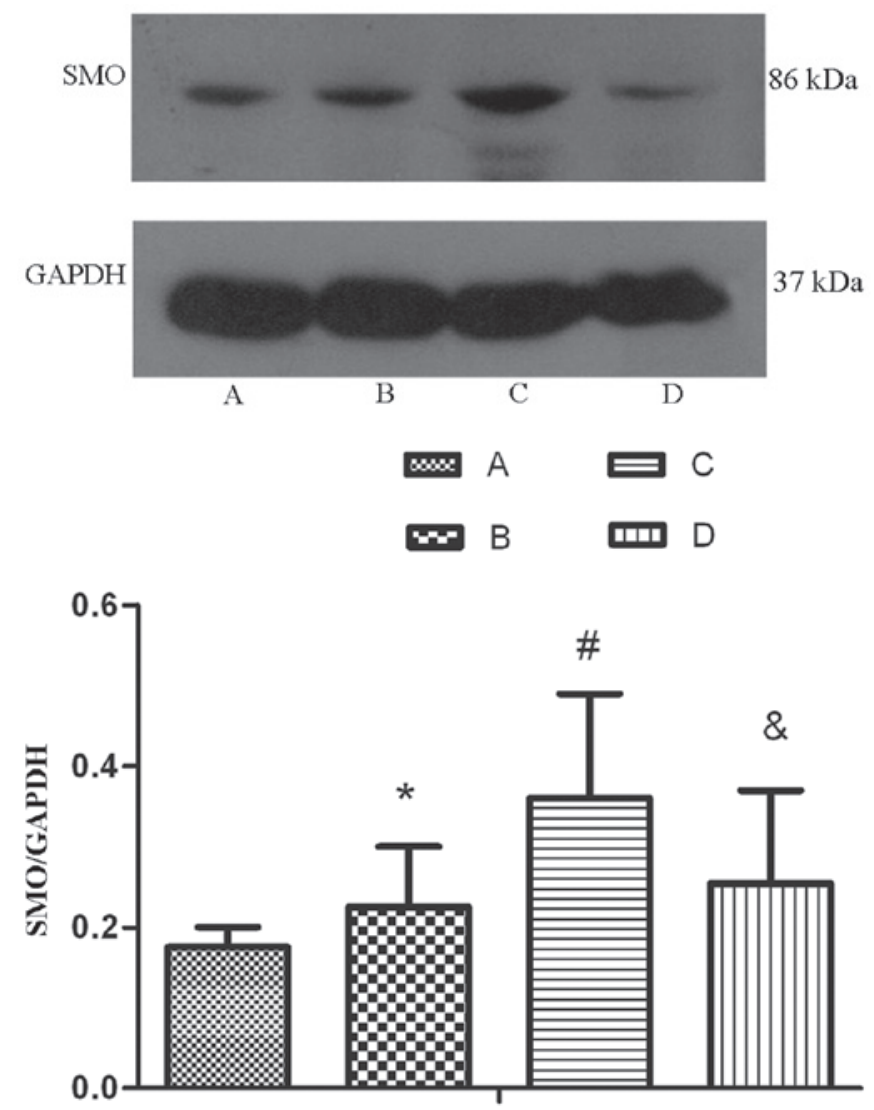

Figure 5. Protein level of SMO in alveolar epithelial type II cells. SMO protein expression was significantly higher in the hyperoxia-exposed group compared with that in the air-exposed group. The expression level of the SMO protein significantly increased in the cells stimulated by SP. The expression of the SMO protein was inhibited by L703.606. A, air group; B, hyperoxia group; C, hyperoxia + SP group; D, hyperoxia + SP + L703.606 group); SMO, Smoothened; SP, substance P; GAPDH, glyceraldehyde-3-phosphate dehydrogenase. ${ }^{*} \mathrm{P}<0.05$ vs. $\mathrm{A},{ }^{\#} \mathrm{P}<0.05$ vs. $\mathrm{B}$ and ${ }^{\star} \mathrm{P}<0.05$ vs. $\mathrm{C}$.

sion of SMO proteins in the hyperoxia-exposed group was elevated by stimulation with SP. SP was able to significantly increase the expression of SMO. The upregulation effect aforementioned was attenuated following treatment with L703.606 (Fig. 5).

\section{Discussion}

Hyperoxia-induced lung injury is considered to be a bimodal process resulting from direct oxygen toxicity and the accumulation of inflammatory mediators within the lungs (12). Either reducing lung oxidative stress injury or improving the survival of alveolar epithelial cells may be a way to ameliorate or prevent hyperoxia-induced lung injury. To date, however, no therapy has been identified to potently or consistently prevent or reverse hyperoxia-induced lung injury. AEC IIs may serve as 'alveolar stem cells' (13) and, consequently, the protection of type II epithelial cells during oxygen therapy may be the key to preventing hyperoxia-induced lung injury.

SP is an important sensory neuropeptide. Subsequent to its release, SP binds primarily to NK-1 receptors (NK-1R) regulating airway smooth muscle responses, airway inflammation, and epithelial migration and proliferation $(14,15)$. Oslund et al (16) found that SP is an important mediator in airway epithelial cell death and subsequent proliferation following ozone exposure. Scott et al (5) revealed that SP levels were greater in hypertrophic scars than in uninjured skin, and may result in an exuberant neuroinflammatory response, which is associated with cell proliferation and regeneration. Yaraee et al (17) found that SP was able to directly modulate the release of TGF- $\beta$ from the human bronchial epithelial cell line and thereby is involved in various lung functions or pathological conditions. Dib et al (18) found that sensory neurotransmitters were able to protect acute hyperoxic lung injury from aggravation by activating NK1R-mediated functions.

The present study demonstrated that AEC IIs exposed to $95 \%$ oxygen for $24 \mathrm{~h}$ may cause cellular injury, increase the production of ROS, induce apoptosis and inhibit cellular proliferation. However, SP was able to decrease the inhibition of hyperoxia on cell proliferation, reduce AEC II apoptosis and necrosis, and improve cell survival sequentially. In addition, the aforementioned protective effect was attenuated following treatment with L703.606. The data suggested that SP was able to decrease oxidative damage in AEC IIs and that SP interference may be a protective strategy for AEC IIs under oxidative stress. In our previous study, SP protected against hyperoxia-induced lung damage by upregulating the SHH signaling pathway (19).

Information has emerged regarding the mechanisms and significance of the SHH-SMO-Gli pathway in lung morphogenesis. Bellusci et al (20) found that the SHH signaling pathway is important in lung morphogenesis and $\mathrm{SHH}$ overexpression in transgenic mice results in increased mesenchymal and epithelial cell proliferation. Litingtung et al (21) reported that $\mathrm{SHH}$-deficient mice resulted in a phenotype with defects in tracheoesophageal and lung morphogenesis, including hypoplastic lung buds, loss of lung symmetry and a single tracheoesophageal tube. Pepicelli et al (22) also found that the ectopic expression of SHH increases proliferation and in SHH null mutants, the trachea and esophagus do not separate properly and the lungs form a rudimentary sac. Overexpression of $\mathrm{SHH}$ in the lung results in increased levels of Gli mRNA and GLI1 is the principal effector of SHH signaling (23). It has been demonstrated that initiation of lung morphogenesis is instructed by SHH signaling (24).

In order to elucidate the effects of SP on the $\mathrm{SHH}$ signaling pathway, the mRNA and protein expression levels of SMO were determined. The results from the present study demonstrated that exposure to hyperoxia significantly activated SMO transiently and supplementary SP improved cell survival and decreased apoptosis accompanied by enhancing the expression of SMO. To further confirm that the activation of the SHH pathway was associated with the protection cells against hyperoxia by SP, L703.606 was added. The cells in the L703.606 group showed increased ROS activity, enhanced apoptosis and decreased proliferation, accompanied by the decreasing expression of SMO, compared with the SP group.

It is suggested that SP interference, a protective regulatory factor, had a protective effect on AEC IIs exposed to hyperoxia, which may be associated with the promotion of the activation of SHH pathways. SP may therefore have a therapeutic effect on patients with hyperoxia-induced lung injury. 


\section{Acknowledgements}

This study was supported by the National Natural Science Foundation of China (no. 30973218).

\section{References}

1. Gordo-Vidal F, Calvo-Herranz E, Abella-Alvarez A, et al: Hyperoxia-induced pulmonary toxicity. Med Intensiva 34: 134-138, 2010 (In Spanish).

2. Buccellato LJ, Tso M, Akinci OI, et al: Reactive oxygen species are required for hyperoxia-induced Bax activation and cell death in alveolar epithelial cells. J Biol Chem 279: 6753-6760, 2004.

3. Miyake Y, Kaise H, Isono K, et al: Protective role of macrophages in noninflammatory lung injury caused by selective ablation of alveolar epithelial type II Cells. J Immunol 178: 5001-5009, 2007.

4. Scott JR, Muangman P and Gibran NS: Making sense of hypertrophic scar: a role for nerves. Wound Repair Regen 15 (Suppl 1): S27-S31, 2007.

5. Scott JR, Muangman PR, Tamura RN, et al: Substance P levels and neutral endopeptidase activity in acute burn wounds and hypertrophic scar. Plast Reconstr Surg 115: 1095-1102, 2005.

6. Zhang M, Wang H, Teng H, et al: Expression of SHH signaling pathway components in the developing human lung. Histochem Cell Biol 134: 327-335, 2010.

7. Li Y, Zhang H, Choi SC, et al: Sonic hedgehog signaling regulates Gli3 processing, mesenchymal proliferation, and differentiation during mouse lung organogenesis. Dev Biol 270: 214-231, 2004

8. Le H, Kleinerman R, Lerman OZ, et al: Hedgehog signaling is essential for normal wound healing. Wound Repair Regen 16 : 768-773, 2008

9. Luo JD, Hu TP, Wang L, et al: Sonic hedgehog improves delayed wound healing via enhancing cutaneous nitric oxide function in diabetes. Am J Physiol Endocrinol Metab 297: e525-e531, 2009.

10. Kusano KF, Pola R, Murayama T, et al: Sonic hedgehog myocardial gene therapy: tissue repair through transient reconstitution of embryonic signaling. Nat Med 11: 1197-1204, 2005.
11. Corti M, Brody AR and Harrison JH: Isolation and primary culture of murine alveolar type II cells. Am J Respir Cell Mol Biol 14: 309-315, 1996.

12. Pagano A and Barazzone-Argiroffo C: Alveolar cell death in hyperoxia-induced lung injury. Ann N Y Acad Sci 1010: 405-416, 2003.

13. Bishop AE: Pulmonary epithelial stem cells. Cell Prolif 37: 89-96, 2004.

14. Hökfelt T, Pernow B and Wahren J: Substance P: a pioneer amongst neuropeptides. J Intern Med 249: 27-40, 2001.

15. Hafstrom I, Ringertz B, Lundeberg T and Palmblad J: The effect of endothelin, neuropeptide Y, calcitonin gene-related peptide and substance $\mathrm{P}$ on neutrophil functions. Acta Physiol Scand 148: 341-346, 1993.

16. Oslund KL, Hyde DM,Putney LF, et al: Activation of neurokinin-1 receptors during ozone inhalation contributes to epithelial injury and repair. Am J Respir Cell Mol Biol 39: 279-288, 2008

17. Yaraee R and Ghazanfari T: Substance P potentiates TGF $\beta-1$ production in lung epithelial cell lines. Iran J Allergy Asthma Immunol 8: 19-24, 2009.

18. Dib M, Zsengeller Z, Mitsialis A, et al: A paradoxical protective role for the proinflammatory peptide substance $\mathrm{P}$ receptor (NK1R) in acute hyperoxic lung injury. Am J Physiol Lung Cell Mol Physiol 297: L687-L697, 2009.

19. Yang L, Liu C, Dang H, et al: Substance $P$ attenuates hyperoxia-induced lung injury in neonatal rats. Mol Med Rep 9: 595-599, 2014.

20. Bellusci S, Furuta Y, Rush MG, et al: Involvement of Sonic hedgehog (Shh) in mouse embryonic lung growth and morphogenesis. Development 124: 53-63, 1997.

21. Litingtung Y, Lei L, Westphal $\mathrm{H}$ and Chiang C: Sonic hedgehog is essential to foregut development. Nat Genet 20: 58-61, 1998.

22. Pepicelli CV, Lewis PM and McMahon AP: Sonic hedgehog regulates branching morphogenesis in the mammalian lung. Curr Biol 8: 1083-1086, 1998.

23. Grindley JC, Bellusci S, Perkins D and Hogan BL: Evidence for the involvement of the Gli gene family in embryonic mouse lung development. Dev Biol 188: 337-348, 1997.

24. Warburton D, Zhao J, Berberich MA, et al: Molecular embryology of the lung: then, now, and in the future. Am J Physiol 276 : L697-L704, 1999. 